\title{
The Application of Agents to Parallel Mesh Refinements in Domain Decomposition Based Parallel Fully Automatic $h p$ Adaptive Finite Element Codes
}

\author{
Maciej Paszynski \\ AGH University of Science and Technology, \\ Al. Mickiewicza 30, 30-059 Cracow, Poland \\ paszynsk@agh.edu.pl \\ http://home.agh.edu.pl/ paszynsk
}

\begin{abstract}
In the $h p$ adaptive Finite Element Method (FEM) applications, the computational mesh consists in finite elements with varying size $h$, and varying polynomial order of approximation $p$ on finite element edges, faces and interiors. The parallel $h p$ adaptive codes work on the computational domain partitioned into sub-domains with each of the sub-domains delegated to a single processor. The algorithm of parallel mesh refinements on such a distributed FE must enforce global mesh regularity rules. The paper presents the applications of multiple agents to implement the parallel mesh refinements algorithm. Agents work on distributed data structure storing FE mesh where dynamic mesh refinements are recorded by growing trees of initial mesh elements nodes. Agents located on separated sub-domains communicate in order to establish necessary actions on the distributed mesh.
\end{abstract}

\section{Introduction}

Parallelization of $h$ or $h p$ adaptive codes is difficult. Most implementations for distributed memory parallel computers are based on Domain Decomposition (DD) paradigm. The computational domain is partitioned into sub-domains with each of the sub-domains assigned to a single processor.

Among major undertakings to develop a general infrastructure to support DD based parallelization of PDE solvers, one has to list first of all the Sierra Environment [6] developed by Sandia National Labs, supporting h-adaptivity with polynomial order of approximation $p=2$. The Sierra environment allows for an arbitrary domain partitioning of a current mesh but it does not support anisotropic mesh refinements. General environments to support local mesh refinements have been developed by [3, 7] and, more recently, [13. None of them, unfortunately, turned out to be applicable directly to our $h p$ codes. The only parallel $h p$ codes that we are aware of, have been developed by [12, [9], [2] and [1]. None of them are fully automatic hp adaptive codes. The parallel fully automatic $h p$ adaptive 2D and 3D FE codes were finally implemented by the group of Demkowicz [11,10, based on the data structures described in [5]. 
The paper describes applications of agents to parallel mesh refinements performed during iterations of automatic $h p$ adaptive $2 \mathrm{D}$ and $3 \mathrm{D}$ FE codes. Agents work on distributed data structure storing FE mesh where dynamic mesh refinements are recorded by growing trees of initial mesh elements nodes. Agents located on separated nodes communicate to enforce global mesh regularity rules which must be fulfilled during refinements on distributed mesh.

\section{The Parallel Fully Automatic $h p$ Adaptive Algorithm}

Let us focus on the 3D Fichera model problem, presented in Figure 1, described in [10]. The problem consists in solving the Laplace equation on the $3 \mathrm{D}$ cube with $1 / 8$ smaller cube removed, see picture (a). The Neumann boundary conditions based on the known exact solution is applied on the external boundary of the cube, denoted by the red color. Zero Dirichlet boundary condition is applied on the internal boundary, denoted by the blue color. There are strong singularities in the middle point of the cube, as well as along edges of removed smaller cube. Those singularities produce large numerical error of the solution. To reduce the error, automatic $h p$ adaptive strategy enforces optimal mesh refinements in those areas.

The parallel strategy starts from the coarse mesh, redistributed into processors, as it is presented in picture (b). In the example 7 processors were utilized. The coarse mesh is then globally $h p$ refined. Each element from the coarse mesh is broken into 8 sons in 3D, and the order of approximation is uniformly risen by one, see picture (c). The problem is solved twice, on the coarse and on the fine mesh. For each finite element from the coarse mesh we consider various refinement strategies, and evaluate error decrease rate based on comparison of coarse and fine grid solutions. Optimal refinement is selected for each finite element from the coarse mesh. The optimal mesh after the first iteration is obtained by performing optimal mesh refinements on the coarse mesh by the parallel mesh refinements algorithm, see picture (d). The mesh must be globally consistent, which requires involving of mesh reconciliation algorithms in the parallel mesh refinements algorithm. The computational load over the optimal mesh is not well balanced, since the refinements are not uniform. There is a need to balance load, by removing some finite elements from some processors and sending them to some neighboring processors. The ZOLTAN load balancing library [14 is utilized to obtain optimal load distribution. The optimal mesh redistributed onto 7 processors becomes the coarse mesh for the second iteration, see picture (e). The mesh is again globally $h p$ refined, see picture (f). The problem is again solved twice on the coarse and fine mesh, and the optimal refinements are selected for the coarse mesh, based on relative error estimations over each finite element from the coarse mesh. Finally optimal mesh refinements are executed by parallel mesh refinements algorithms, see picture (g). The iterations are performed as long as the estimated error is large. Optimal mesh obtained after 5 iterations providing solution to the Fichera problem with $1 \%$ relative error in energy norm is presented in pictures (h) and (i). 


\section{Distributed Data Structure for Finite Element Mesh Supporting $h p$ Adaptivity}

In the data structure for distributed storage of FE mesh [5] only initial mesh elements are stored. When a finite element is broken into new son elements, refinement trees grows from initial mesh elements nodes, and newly created nodes and vertices are stored on these trees, see Figure 2. Each initial mesh element in $3 \mathrm{D}$ consists in 8 vertices, 12 mid-edge nodes, 6 mid-face nodes and 1 middle node. The element structure keeps pointers to all its vertices and nodes, pointers to 6 neighbors (initial mesh elements), orientation and boundary condition data. All of the vertices have polynomial order of approximation fixed to one. A finite element can be broken into 2, 4 or 8 new son elements. A face of an element is broken only if all neighboring elements are broken, including elements located on adjacent sub-domains. An edge of an element is broken only if all faces neighboring the edge are broken, including faces located on neighboring sub-domains. An element interior can be always broken. Newly created finite elements are not stored in the data structure.

To break an edge means to create 2 new mid-edge nodes and 1 new vertex and attached them as sons of the original mid-edge node. The refinement tree grows down from the mid-edge node. A face can be broken into 2 or 4 faces. To break a face into 2 new faces means to create 2 new mid-face nodes and 1 new mid-edge node. To break a face into 4 new faces means to create 4 new mid-face nodes, 4 new mid-edge nodes and 1 new vertex node. A middle node representing an element interior can be broken into 2,4 or 8 sons. To break an element interior into 2 sons means to create 2 new middle nodes and one new mid-face node. To break an element interior into 4 sons means to create 4 new middle nodes, 4 new mid-face nodes and one new mid-edge node. To break an element into 8 sons means to create 8 new middle nodes, 12 new mid-face nodes, 6 new mid edge nodes and one new vertex node.

Each node keeps its polynomial order of approximation, type (mid-edge, midface or middle node), finite element degrees of freedom data, and the refinement tree data, involving: lists of sons, refinement type of the node, and a pointer to the father. Number and type of sons on the list depends on the node refinement type. Finite element method computations are performed on active elements. Their nodes and vertices are found dynamically by browsing the refinement trees from the level initial mesh elements.

\section{Agent Based Algorithm for Parallel Mesh Refinements}

In the parallel mesh refinement algorithm some finite elements are divided into multiple (new) elements (each rectangular element is divided into 2 or 4 sons in $2 \mathrm{D}$ and 2,4 or 8 sons in 3D), new nodes are created, and new orders of approximation are set. The parallel version of the algorithm requires an enforcement of a global mesh regularity rules: 


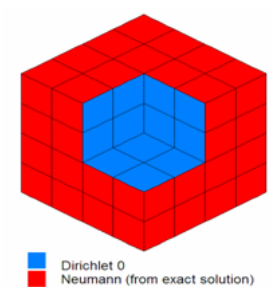

(a)

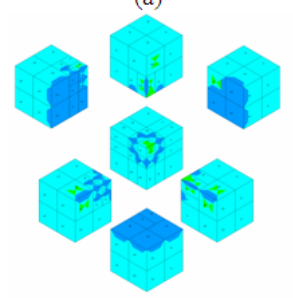

(d)

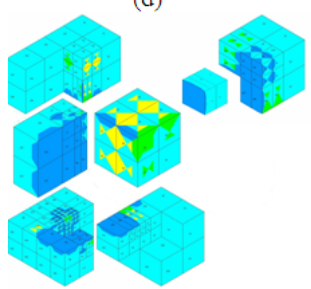

(g)

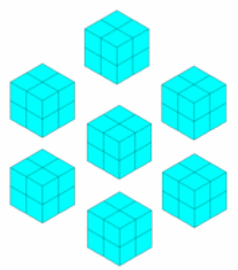

(b)

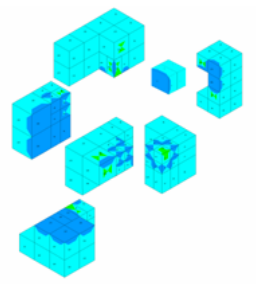

(e)

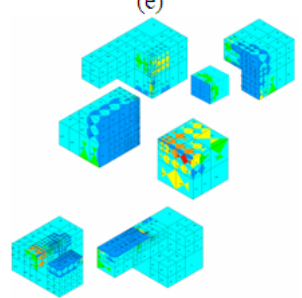

(h)

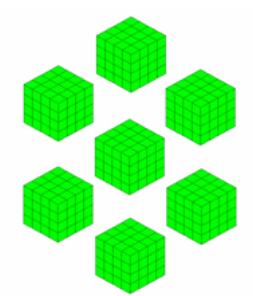

(c)

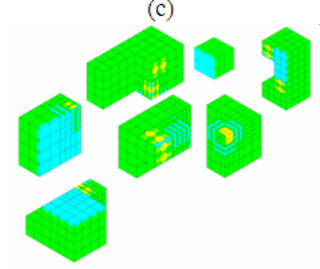

(f)

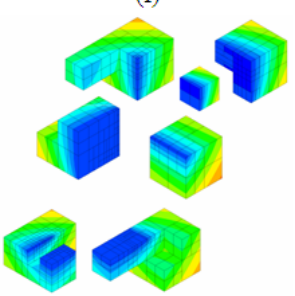

(i)

Fig. 1. Parallel fully automatic $h p$ adaptive iterations
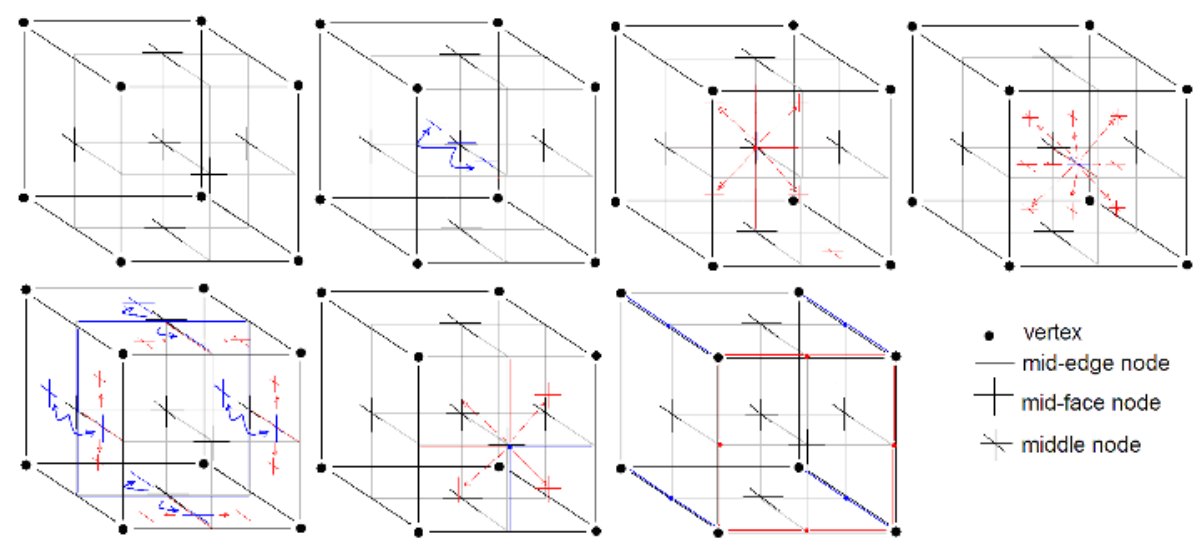

\section{- vertex \\ + mid-edge node \\ t middle node}

Fig. 2. Breaking an element into 2 sons, followed by breaking of one son element into 4 new son elements. The blue color denotes all refinement trees created during the first refinement, and the red color denotes refinement trees created during the second refinement. We assume that all affected element's faces and edges can be broken. 
- The 1-irregularity rule: Edges of a given element can be broken only once, without breaking neighboring elements. It is necessary to check the configuration of elements adjacent on neighboring sub-domains to the element requested to be broken.

- When an interface edge is broken, then the polynomial order of newly created nodes must be established. The selection of optimal order for a node is done based on error estimations performed over the finite elements having that node. But some of the elements neighboring the interface edge are present in adjacent sub-domains. It is necessary to ask the neighboring sub-domains for the error estimation over those elements.

- The minimum rules: Order of approximation of an element interior cannot be larger then orders of approximation of the element faces, and order of approximation of a face cannot be larger then orders of approximation of faces adjacent to the edge. To enforce the minimum rule, we need to exchange orders of approximation for interface faces and edges with adjacent sub-domains.

We will discuss now the applications of agents to particular problems appearing during refinements on distributed mesh. The agents work on distributed data structure storing the mesh with refinements coded as growing trees of initial mesh elements nodes.

\section{Enforcing 1-irregularity rule}

The 1-irregularity rule requires breaking of neighboring elements if the current element must be broken for the second time. There is a need to send a request to adjacent sub-domains to break neighboring elements. The way of breaking the neighboring elements depends on the configuration of the current and the neighboring elements.

The refinement on current sub-domain is performed by an agent, that is responsible for enforcing mesh regularity rules. If the element was previously broken, the agent responsible for managing breaking the element on current sub-domain packs the information about the previous refinement of the element. The current element could be previously broken in one of many possible ways: the element could be broken into 2 sons in one of 3 possible directions, the element could be broken into 4 sons in one of 3 possible directions, or the element could be broken into 8 sons. The refinement trees have different syntax in all those cases. The agent must communicate with the agent located in other location, and request necessary refinements on the neighboring elements. The agent must pack the refinement tree and send it to agents located in adjacent sub-domains. The refinement trees are then unpacked by the agents located on adjacent sub-domains, and the decision about kind of necessary refinements of the adjacent elements are made by the remote agents by comparing received and local refinement trees.

\section{Breaking of an element neighboring the interface}

Breaking of an element involves the following steps: breaking the element interior, breaking the element faces and breaking the element edges. The element interior can be always broken. The element face can be broken only if interior of the 
element neighboring current element possibly on adjacent sub-domain is broken, or the element is adjacent to the boundary of the domain. The element edge can be broken only if all faces adjacent to the edge are broken, including faces located on current and adjacent sub-domains.

\section{Breaking of an interface face}

It is necessary to ask elements located on adjacent sub-domain if its interior is broken. The agent responsible for breaking an interface face must send the question to remote agent located on adjacent sub-domain. The remote agent locates the initial mesh element containing the neighboring element on adjacent sub-domain by using the connectivity information stored in the initial mesh elements. The neighboring element is found by traveling down the refinement tree in the neighboring initial mesh element.

\section{Breaking of an interface edge}

It is necessary to locate all elements neighboring the edge located on adjacent sub-domains, and check if faces of these elements adjacent to the edge are broken. The agent responsible for breaking an interface edge must communicate with all agents located in adjacent sub-domains and request a check of state of their faces. The location of neighboring elements can be found by using the connectivity information stored in the initial mesh elements.

\section{Setting orders of approximation for nodes located on the interface}

In the first step of the fully automatic $h p$ adaptive algorithm, optimal $h$ refinements are performed, by breaking appropriate middle nodes, mid-face nodes and mid-edge nodes. In the next step of the algorithm, decisions about optimal orders of approximation are made. In the first version of the automatic $h p$ adaptivity [4, 5, 11], decisions about optimal orders of approximations were made for edges, faces and interiors. Those decisions were made based on error estimations computed on current and neighboring elements. It was necessary to exchange information about error estimations on neighboring elements located on adjacent sub-domains. In the new version of the algorithm [8,10, decisions about optimal order of approximation are made only for element interiors. The error estimation for the element is computed by using only local information contained in the element under consideration. The operation is fully parallel. The orders of approximation for faces and edges are computed by enforcing minimum rule.

\section{Enforcing the minimum rule for interface edges and faces}

In its first step, the minimum rule requires to adjust orders of approximation of faces as a minimum of orders of approximation of interiors of neighboring elements, possibly located on adjacent sub-domains. The agents exchange information about the orders of approximation of interiors of elements neighboring the interface between adjacent sub-domains. Then, the orders of faces located on the interface can be set by agents based on the obtained orders of approximations of elements interiors from adjacent sub-domains. The neighboring elements can be found by using connectivity information stored in the initial mesh elements. In its second step, the minimum rule requires to adjust orders of approximation 


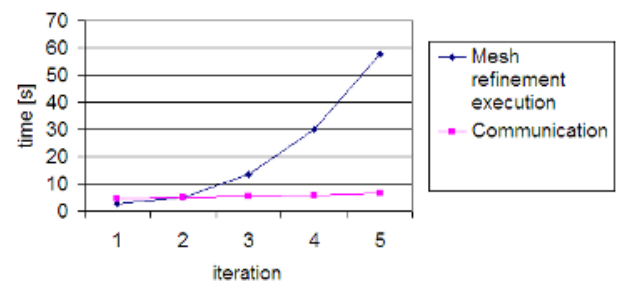

Fig. 3. Measurements of times of execution of mesh refinements versus communication

of mid-edge nodes as a minimum of orders of neighboring faces, possibly located on adjacent sub-domains. The agents exchange information about the orders of approximation of faces of elements neighboring the interface. Then, the orders of edges located on the interface can be set by agents based on the obtained orders of approximations of faces from adjacent sub-domains.

\section{Performance test}

In the parallel fully automatic $h p$ adaptive codes [11, [10 there is a module responsible for execution of mesh refinements on the distributed data structure. It is like copies of identical agents, assigned one to each sub-domain, working in concurrent with the rest of the parallel code. Each agent executes on its subdomain all mesh refinements that can be performed without communication with adjacent agents, and collects data about refinements on the interface that need to be exchanged with adjacent agents. Then, all agents exchange data according to coloring of edges of created communication graph. The algorithm is repeated unless no exchange of data with neighboring sub-domains is needed (unless the interface is not affected). It follows that the communication takes less time than execution of mesh refinements, see Figure 3. The mesh refinements execution time can be reduced by increasing number of agents working in cuncurrent on each sub-domain. Thus, the application of many concurrently working agents may improve the scalability of the code.

\section{Conclusions}

The parallel mesh refinements algorithm can be efficiently performed on the distributed data structure where the refinements are recorder by growing trees of initial mesh elements nodes. The algorithm can be realized by a set of agents working on the distributed data structure. There are two possible solutions. In the first approach each agent can be assigned to one sub-domain, and it can share its work to perform its own tasks with tasks requested by neighboring agents. This is the solution we use in our parallel codes. In the second solution, each agent can remotely invoke agents in other locations to execute its tasks on remote piece of the mesh. In such a case many agents can perform their tasks in concurrent on each sub-domain. The second solution may be more efficient, however it requires to implement synchronization mechanisms to ensure that the data structure won't be damaged by many concurrently working agents. 


\section{Acknowledgement}

The support of this work under MEiN grant no. 3 T08B 05529 is gratefully acknowledged.

\section{References}

1. Banaś K.: A Model for Parallel Adaptive Finite Element Software, Proceedings of 15th International Conference on Domain Decomposition Methods, Freie Universitat Berlin, July 21-25 (2003)

2. Bastian P., Birken K., Johannsen K., Lang S., Neuss N., Rentz-Reichert H., and Wieners C.: UG - a flexible software toolbox for solving partial differential equations. Computing and Visualization in Science, 1, 1, (1997) 27-40

3. Das R., Hwang Y.-S., Uysal M., Saltz J., Sussman A.: Applying the CHAOS/PARTI Library to Irregular Problems in Computational Chemistry and Computational Aerodynamics. Proceedings of the Scalable Parallel Libraries Conference. Mississippi State University, Starkville, (1993) 45-46

4. Demkowicz L.: 2D hp-Adaptive Finite Element Package (2Dhp90) Version 2.0. TICAM Report 02-06 (2002)

5. Demkowicz L., Pardo D., Rachowicz W.: 3D hp-Adaptive Finite Element Package (3Dhp90) Version 2.0, The Ultimate (?) Data Structure for Three-Dimensionsl, Anisotropic $h p$ Refinements, TICAM Report 02-24. (2002)

6. Edwards H. C.: Sierra Framework for Parallel Adaptive Multiphisics Computational Mechanics Applications. VIII US National Congress on Computational Mechanics, Austin, TX. (2005)

7. Fink S.J., Kohn S.R., Baden S.B.: Efficient run-time support for irregular blockstructured applications. J. Parallel Distrib. Comput., 50, 1-2, (1998) 61-82

8. Kurtz J.: Fully Automatic $h p$-Adaptivity for Acoustic and Electromagnetic Scattering in Three Dimensions. Dissertation Proposal. The University of Texas at Austin. (2005)

9. Laszloffy A., Long J., Patra A. K.: Simple data management, scheduling and solution strategies for managing the irregularities in parallel adaptive hp finite element simulations. Parallel Computing, 26, (2000) 1765-1788

10. Paszyński M., Demkowicz L.: Parallel Fully Automatic $h p$-Adaptive 3D Finite Element Package. ICES Report 05-33. VIIIth U.S. National Congress on Computational Mechanics, Austin, Texas. submitted to Engineering with Computers (2005)

11. Paszyński M., Kurtz J., Demkowicz L.: Parallel Fully Automatic $h p$-Adaptive 2D Finite Element Package. ICES Report 04-07. Computer Methods in Applied Mechanics and Engineering, 195, 7-8, (2006) 711-741

12. Remacle J. F., Xiangrong Li, Shephard M. S., Flaherty J. E.: Anisotropic Adaptive Simulations of Transient Flows using Discontinuous Galerkin Methods. Int. J. Numer. Meth. Engng. 62, 7, (2005) 899-923

13. Yelick K. et al.: Titanium: A High-Performance Java Dialect. ACM 1998 Workshop on Java for High-Performance Network Computing, Stanford, California, February (1998)

14. ZOLTAN: Data Management Services for Parallel Applications, http://www.cs.sandia.gov/zoltan 